\title{
Value of intracellular bacteria detection in the diagnosis of ventilator associated pneumonia
}

\author{
A Torres, M El-Ebiary, N Fábregas, J González, J Puig de la Bellacasa, C Hernández, \\ J Ramírez, R Rodriguez-Roisin
}

\begin{abstract}
Background - Markers of ventilator associated pneumonia are of interest for confirming the diagnosis and for guiding the initial management of this frequent complication of mechanical ventilation. The detection of intracellular organisms in the polymorphonuclear leucocytes (PMNLs) and/or macrophages of bronchoalveolar lavage (BAL) fluid has been suggested as a specific test for the early indication of an infectious pulmonary process.
\end{abstract}

Methods - The diagnostic value of detecting intracellular organisms in two types of BAL fluid - protected (P-BAL) and conventional (C-BAL) - in 25 patients who died in one unit was prospectively studied. Immediately after death both PBAL and C-BAL were performed bilaterally. Through a minithoracotomy on both sides of the chest bilateral bronchoscopically guided open lung biopsy samples were obtained from the same area, and an average of eight open lung blind biopsy samples (not bronchoscopically guided) were taken from each lung for histological examination. BAL fluid was examined for quantitative cultures (threshold $10^{4} \mathrm{cfu} / \mathrm{ml}$ ) and for the presence of intracellular organisms and extracellular organisms, and differential cell counts were also performed.

Servei de Pneumologia i Al.lèrgia Respiratòria A Torres

M El-Ebiary

C Hernández

R Rodriguez-Roisin

Servei de

Anestesiologia

N Fábregas

Servei de

Microbiologia

J González

J Puig de la Bellacasa

Servei de Anatomia Patologica

J Ramírez

Departament de Medicina,

Hospital Clínic, Universitat de

Barcelona,

Villarroel 170, 08036

Barcelona, Spain

Correspondence to: Dr A Torres.

Received 18 May 1995 Returned to authors

30 August 1995

Revised version received

25 September 1995

Accepted for publication

Accepted for publicat discriminate between the presence or absence of pneumonia.

Conclusions - The presence of $\geq 5 \%$ intracellular organisms infecting PMNLs or macrophages in P-BAL or C-BAL fluids is a specific marker of ventilator associated pneumonia.

(Thorax 1996;51:378-384)

Keywords: intracellular organisms, protected bronchoalveolar lavage, ventilator associated pneumonia.

Ventilator associated pneumonia occurs in $9-70 \%$ of patients who need mechanical ventilation ${ }^{12}$ and accounts for one third of the overall mortality of patients who develop it. ${ }^{3}$ However, the optimal technique for diagnosing nosocomial bacterial pneumonia in patients receiving mechanical ventilation remains elusive since new information ${ }^{4}$ suggests that noninvasive methods could suffice for the clinical management of these patients. The problem with quantitative cultures of any technique, invasive or non-invasive, which samples airways is that cultures take 2-4 days to be of value. Accordingly, critical decisions concerning antibiotic treatment may be delayed. The use of specific markers of ventilator associated pneumonia - namely, the presence of intracellular microorganisms (intracellular organisms), ${ }^{5}$ the levels of endotoxins ${ }^{6}$ or lactate dehydrogenase in the bronchoalveolar lavage (BAL) fluid, and the detection of elastin fibres ${ }^{8}-$ may be of value as they can provide a rapid diagnosis of ventilator associated pneumonia and can be of help in the initial decision of choosing an antibiotic regimen. Of these markers, the presence of intracellular organisms in BAL fluid appears to be the most specific. ${ }^{5}$ However, few studies have reported the diagnostic value of this method in mechanically ventilated patients with ventilator associated pneumonia. ${ }^{9-12} \mathrm{We}$ have therefore performed a prospective study to determine the value of intracellular organisms in the BAL fluid for diagnosing ventilator associated pneumonia using the histological results of immediate necroscopic pulmonary biopsy samples as the gold standard.

\section{Methods}

PATIENTS

Over a one year period 25 patients who died in our respiratory intensive care unit after more than 72 hours of mechanical ventilation were included in the study. Patients with immunosuppression or haematological malignancies were excluded. General characteristics of the study population are described in table 1 . Seventeen patients received prior antibiotic therapy while the remaining eight had not received antibiotics for at least 48 hours before the start of the study. Those who had had antibiotics 
Table 1 . Mean (SD) characteristics of the study population

\begin{tabular}{lc}
\hline Age (years) & $56 \cdot 7(16)$ \\
M/F & $16 / 9$ \\
APACHE II before death & $27(6)$ \\
Temperature $\left({ }^{\circ} \mathrm{C}\right)$ & $36(1)$ \\
Leucocyte count $\left(\times 10^{9} / 1\right)$ & $14 \cdot 7(8 \cdot 4)$ \\
$\mathrm{PaO}_{2} /$ FiO & \\
$\mathrm{Mechanical} \mathrm{ventilation} \mathrm{period} \mathrm{(days)}_{\text {No. of antibiotics }}$ & $207(126)$ \\
Duration of antibiotic treatment (days) & $13(13)$ \\
Days without antibiotics $(\mathrm{n}=8)$ & $4(2)$ \\
Underlying conditions: & $9 \cdot 5(7 \cdot 9)$ \\
COPD & $3 \cdot 2(2)$ \\
Liver disease & $7(28 \%)$ \\
Cardiovascular disease & $1(4 \%)$ \\
Collagen disease & $7(28 \%)$ \\
Others & $3(12 \%)$ \\
\hline
\end{tabular}

Values were obtained immediately after death ( \pm 30 minutes $)$ at the time of initiation of the study.

APACHE II = Acute Physiology and Chronic Health Evaluation APACHE $\mathrm{II}=$ Acute Physiology and Chronic Health Evaluation
score; $\mathrm{COPD}=$ chronic obstructive pulmonary disease; $\mathrm{PaO}_{2}=$ arterial oxygen tension; $\mathrm{FiO}_{2}=$ fractional inspired oxygen.

received a mean (SD) of 4 (2) drugs for a mean duration of $9.5(7 \cdot 9)$ days. These included: cefonicid, cefotaxime, ceftazidime, ciprofloxacin, fluconazol, vancomycin, clindamycin, netilmicin, trimethoprim/co-trimoxazole, imipenem, amikacin, and amphotericin B. Patients were considered not to have received antibiotics if they had not been given any for at least 48 hours before death. The administered antibiotics in these patients were: erythromycin, cefonicid, cefotaxime, ciprofloxacin, fluconazol, vancomycin, imipenem, amikacin, and amphotericin B. Causes of death included: cerebral death (12), multiple organ failure (9), and severe hypoxaemia (4). All patients except four had either localised $(n=14)$ or diffuse $(n=7)$ infiltrates on their chest radiographs. The clinical diagnoses of pulmonary infiltrates $(n=21)$ on the day of death were: pneumonia (19), congestive heart failure (1), and both alveolar haemorrhage and pneumonia (1). The percentage of samples showing pneumonia was higher in lung samples from patients who presented with chest radiographic infiltrates $(50 \%$ localised infiltrates and $62 \%$ diffuse infiltrates) than in those with normal chest radiographs $(19 \%)$. The correlation between the percentage of positive lung biopsy samples for histological pneumonia in each patient and the time interval between chest radiographic infiltrate appearance and death was poor $(r=0 \cdot 2, \mathrm{p}=\mathrm{NS})$. The main reasons for admission to the intensive care unit (ICU) were: acute respiratory failure (12), stroke (6), postoperative (3), cranial trauma (3), and myocardial infarction (1). Of the 12 cases of acute respiratory failure six were admitted with pneumonia, two with congestive heart failure, two with alveolar haemorrhage, and two with pulmonary neoplasia. Family members in each case gave informed written consent and permission was obtained from the ethical committee of our centre.

STUDY DESIGN

Immediately after death patients remained mechanically ventilated while breathing $100 \%$ $\mathrm{O}_{2}$. Bilateral fibreoptic bronchoscopies were performed using different bronchoscopes for each lung following standard techniques. The BAL specimens were retrieved in the order: protected bronchoalveolar lavage (P-BAL) (Mill-Rose Laboratories Inc, Ohio, USA) followed by conventional BAL (C-BAL). ${ }^{13}$ Both types of BAL were performed by instilling an aliquot of $20 \mathrm{ml}$ sterile saline which was discarded, followed by four aliquots each of $30 \mathrm{ml}$ sterile saline (mean (SD) recovered BAL fluid 26 (15) $\mathrm{ml}$ and 45 (20) $\mathrm{ml}$ for P-BAL and C-BAL, respectively). Open lung bronchoscopically guided biopsy and lavage samples were taken from the same place - that is, from the area of maximal infiltrates on the chest radiograph or from lower lobes in patients without infiltrates. A total of 94 lavages (47 P$\mathrm{BAL}$ and $47 \mathrm{C}-\mathrm{BAL}$ ) were performed as three lungs from three patients could not be studied.

A minithoracotomy was performed on each side of the chest and a guided biopsy sample was taken from each lung using the light tip of the bronchoscope which was seen shining from within the lung in areas of maximal inflammation as seen on the chest radiograph. If there were no infiltrates on the radiograph the bronchoscope was placed in a lower lobe segment and biopsy samples were taken from that area. In addition, non-guided (blind) open lung biopsies were taken using strict aseptic techniques from each lung through each thoracotomy incision. Three fragments were taken from both upper and lower lobes from each lung and two from the middle lobe and lingula so that the overall number of biopsy samples per patient was 16 (two guided and 14 blind). The total number of biopsies was 375 (in three patients only one lung was sampled). The smallest size of both the bronchoscopically guided and the blind biopsy samples was $2 \times 2 \times 2 \mathrm{~cm}$ each. Each sample was divided into two pieces: one for histological examination and the other for microbiological processing. Pathological criteria for pneumonia included foci with accumulation of polymorphonuclear leucocytes (PMNLs) in the capillaries and adjacent alveolar spaces corresponding to various degrees of evolution and extension. ${ }^{14}$

\section{MICROBIOLOGICAL PROCESSING AND ISOLATE} IDENTIFICATION

Serial dilutions $\left(10^{-1}, 10^{-2}, 10^{-3}\right)$ from each of the P-BAL and C-BAL samples were prepared in sterile normal saline. Biopsy samples were placed in a mortar with sterile sand and $3 \mathrm{ml}$ sterile saline (Vidrafoc, Barcelona, Spain) and homogenised. Serial dilutions $\left(10^{-1}, 10^{-2}\right.$, $10^{-3}$ ) of each sample were prepared in sterile normal saline. One hundred $\mu \mathrm{l}$ of each dilution was inoculated into the following agar media: $5 \%$ sheep blood, chocolate, Center for Disease Control (CDC) blood, Wilkins-Chalgren, McConkey, blood charcoal yeast extract $(B C Y E-\alpha)$, and Sabouraud-dextrose. All cultures were incubated at $37^{\circ} \mathrm{C}$ under aerobic and anaerobic conditions and in $\mathrm{CO}_{2}$ enriched atmosphere. Cultures were evaluated for growth 24 hours and 48 hours later and discarded if negative after five days, except for CDC and Wilkins-Chalgren which were evaluated at seven days and for Sabouraud which was evaluated at four weeks. All micro- 
Table 2 Percentage sensitivity, specificity, positive and negative predictive values (PPV and NPV) of detection of intracellular organisms in BAL fluid (cutoff points $\geq 2 \%, 5 \%$, and 10\%) for diagnosis of ventilator associated pneumonia using bronchoscopically guided open lung biopsy specimen as gold standard

\begin{tabular}{|c|c|c|c|c|c|c|}
\hline & \multicolumn{3}{|c|}{ Without antibiotics } & \multicolumn{3}{|c|}{ With antibiotics } \\
\hline & $2 \%$ & $5 \%$ & $10 \%$ & $2 \%$ & $5 \%$ & $10 \%$ \\
\hline \multicolumn{7}{|c|}{ Protected BAL: } \\
\hline Sensitivity & 75 & $75 \dagger$ & $50 \dagger \ddagger$ & 36 & $28 \ddagger$ & 17 \\
\hline Specificity & 55 & $55 \$$ & $785^{\top}$ & 69 & $77^{\top}$ & 77 \\
\hline PPV & 75 & 75 & 50 & 55 & 57 & 40 \\
\hline NPV & 83 & 83 & 78 & 36 & 37 & 34 \\
\hline \multicolumn{7}{|c|}{ Conventional BAL: } \\
\hline Sensitivity & 75 & $75 \|$ & 50 & 38 & $24 \|$ & 19 \\
\hline Specificity & 44 & $55 \ddagger$ & 55 & 54 & $77 \neq$ & 77 \\
\hline PPV & 37 & 57 & 33 & 57 & $62{ }^{\top}$ & 57 \\
\hline NPV & 80 & 83 & 71 & 35 & 38 & 37 \\
\hline
\end{tabular}

$+p=0.0002 ; \neq p=0.001 ; \oint p=0.0005 ; \| p<0.0001$.

organisms isolated were identified by standard laboratory methods. ${ }^{15}$ Total cell counts of BAL fluid were performed using an aliquot of the original lavage fluid. Preparations were centrifuged with a Cytospin 2 cytocentrifuge (Shandon, Southern Products, UK) and two samples were stained with May-Grünwald Giemsa stain. Differential cell counts were made by examining at least 500 cells. In addition, 300 cells were examined at $1000 \times$ magnification using an oil immersion lens and the percentage of cells containing intracellular organisms per field was determined. A Gram stain of the cytocentrifuged material was made to evaluate the morphological characteristics of the bacteria.

\section{DATA ANALYSIS}

The diagnostic values of the two types of BAL fluid were calculated using $10^{4} \mathrm{cfu} / \mathrm{ml}$ as a cutoff point to differentiate between colonisation and infection. ${ }^{10}$ Diagnostic values of quantitative cultures of both types of BAL samples were calculated excluding yeasts and nonpathogenic staphylococci (Staphylococcus epidermidis). The diagnostic values of intracellular organisms were calculated using different cutoff points $(2 \%, 5 \%$, and $10 \%)$ and analysed statistically using the SPSS/PC + version $4 \cdot 0^{16}$ and the EPIinfo (version 5.01). Results are expressed as mean (SD). Quantitative variables were compared with the Mann-Whitney test and proportions with the $\chi^{2}$ test (Fisher's exact test when needed). Correlations were tested by linear regression analysis. Diagnostic values were calculated and interpreted according to

Table 3 Percentage sensitivity, specificity, positive and negative predictive values (PPV and NPV) of detection of intracellular organisms in BAL fluid (cutoff points $\geq 2 \%, 5 \%$, and 10\%) for diagnosis of ventilator associated pneumonia using blind open lung biopsy specimen as gold standard

\begin{tabular}{|c|c|c|c|c|c|c|}
\hline & \multicolumn{3}{|c|}{ Without antibiotics } & \multicolumn{3}{|c|}{ With antibiotics } \\
\hline & $2 \%$ & $5 \%$ & $10 \%$ & $2 \%$ & $5 \%$ & $10 \%$ \\
\hline \multicolumn{7}{|c|}{ Protected BAL: } \\
\hline Sensitivity & 58 & $58 \dagger$ & 33 & 25 & $18+\ddagger$ & 58 \\
\hline Specificity & 100 & $100^{\circ}$ & 100 & 100 & $100 t$ & 100 \\
\hline PPV & 100 & 100 & 100 & 100 & 100 & 100 \\
\hline NPV & 17 & 17 & 11 & 20 & 21 & 17 \\
\hline \multicolumn{7}{|c|}{ Conventional BAL: } \\
\hline Sensitivity & 67 & $58+$ & 50 & 43 & $25 † \ddagger$ & 21 \\
\hline Specificity & 100 & $100 \dagger$ & 100 & $67 \dagger$ & $83 t^{\top}$ & 83 \\
\hline PPV & 100 & 100 & 100 & 86 & 87 & 86 \\
\hline NPV & 20 & 17 & 14 & 20 & 19 & 18 \\
\hline
\end{tabular}

$\dagger p<0 \cdot 0001 ; \ddagger p=N S$ standard methods. ${ }^{1718}$ Data were analysed using histopathological criteria of pneumonia as the gold standard in two parallel ways: (1) open lung bronchoscopically guided lung biopsies (lung was considered to be positive for pneumonia if the open lung bronchoscopically guided biopsy sample showed histological evidence of pneumonia), and (2) blind open lung biopsies (a lung was considered positive for pneumonia if at least one of the blind open lung biopsy samples showed histopathological evidence of pneumonia). All $p$ values are two tailed and the level of significance was set at $5 \%$.

\section{Results}

INTRACELLULAR MICROORGANISMS, GRAM STAIN, AND CELL COUNTS

In P-BAL samples the mean percentage of intracellular organisms with colony counts of $\geq 10^{4} \mathrm{cfu} / \mathrm{ml}$ was higher than those with cultures yielding $<10^{4} \mathrm{cfu} / \mathrm{ml}$ both in cases that had and had not received antibiotics (23\% (range $0-80$ ) versus $6 \%$ (range $0-90$ ), $\mathrm{p}=\mathrm{NS}$, and $43 \%$ (range $0-90$ ) versus 3\% (range $0-12$ ), $\mathrm{p}<0.05$, respectively). The same was true for C-BAL (36\% (range $0-80$ ) versus 4\% (0-90), $\mathrm{p}=0.0002$, and $49 \%(0-90)$ versus $14 \%(0$ $40), \mathrm{p}<0.05$, respectively).

Quantitative cultures of both P-BAL and CBAL samples showed moderate correlations with the percentages of intracellular organisms found $(r=0.7 ; \mathrm{p}=0.0001 ; r=0.45 ; \mathrm{p}=0.001$, respectively), and these correlations persisted when results from patients who had and had not received antibiotics were compared (PBAL: $r=0.58$ and $r=0.67, \mathrm{p}=0.001$ each; CBAL: $r=0.4$ and $r=0.48, \mathrm{p}=0.01$ each, respectively).

\section{Gold standard: bronchoscopically guided open}

lung biopsies

Cutoff points of $2 \%, 5 \%$, and $10 \%$ were used to evaluate the sensitivity and specificity and positive and negative predictive values of intracellular organisms. Table 2 shows the different diagnostic parameter values for the detection of intracellular organisms in BAL fluid using the bronchoscopically guided pulmonary biopsy as a gold standard. For P-BAL in patients without prior antibiotic treatment, the threshold of $10 \%$ had the most acceptable diagnostic accuracy (sensitivity $50 \%$, specificity $78 \%$ ). However, in patients who had previously received antibiotics a threshold yielding an acceptable diagnostic value was not found. For C-BAL samples the best threshold was 5\% when samples from patients who had not received antibiotics were analysed (sensitivity $75 \%$, specificity $55 \%$ ). Again, no acceptable ratio of sensitivity/specificity was found for the threshold of intracellular organisms giving an acceptable diagnostic value in patients who had been treated with antibiotics. There were no differences in the diagnostic value parameters of detection of intracellular organisms between P-BAL and C-BAL samples. 
Table 4 Mean (SD) cell counts and numbers of extracellular microorganisms obtained from BAL fluid in the diagnosis of ventilator associated pneumonia using bronchoscopically guided open lung pulmonary biopsy specimens as gold standard

\begin{tabular}{|c|c|c|c|c|c|c|c|c|}
\hline & \multicolumn{4}{|c|}{ Samples with pneumonia $(n=25)$} & \multicolumn{4}{|c|}{ Samples without pneumonia $(n=22)$} \\
\hline & \multicolumn{2}{|c|}{$\begin{array}{l}\text { With antibiotics } \\
(n=21)\end{array}$} & \multicolumn{2}{|c|}{$\begin{array}{l}\text { Without antibiotics } \\
(n=4)\end{array}$} & \multicolumn{2}{|c|}{$\begin{array}{l}\text { With antibiotics } \\
(n=13)\end{array}$} & \multicolumn{2}{|c|}{$\begin{array}{l}\text { Without antibiotics } \\
(n=9)\end{array}$} \\
\hline & $P-B A L$ & $C-B A L$ & $P-B A L$ & $C-B A L$ & $P-B A L$ & $C-B A L$ & $P-B A L$ & $C-B A L$ \\
\hline $\begin{array}{l}\text { Polymorphonuclear } \\
\text { leucocytes }\end{array}$ & $63(24)$ & $74(4)$ & $60(27)$ & $66(26)$ & $57(22)$ & $64(24)$ & $55(29)$ & $65(20)$ \\
\hline $\begin{array}{l}\text { Macrophages } \\
\text { Lymphocytes } \\
\text { Extracellular } \\
\text { microorganisms }\end{array}$ & $\begin{array}{c}35(25) \\
2(3) \\
3(4)\end{array}$ & $\begin{aligned} 29 & (21) \\
2 & (2) \\
7 & (12)\end{aligned}$ & $\begin{aligned} 31 & (22) \\
4 & (6) \\
7 & (12)\end{aligned}$ & $\begin{aligned} 27 & (20) \\
2 & (3) \\
3 & (5)\end{aligned}$ & $\begin{array}{c}39(26) \\
1(2) \\
2(1)\end{array}$ & $\begin{aligned} 27 & (22) \\
1 & (2) \\
4 & (7)\end{aligned}$ & $\begin{array}{c}41(28) \\
2(2) \\
3(6)\end{array}$ & $\begin{array}{c}33(18) \\
2(2) \\
3(6)\end{array}$ \\
\hline
\end{tabular}

$\mathrm{P}-\mathrm{BAL}=$ protected bronchoalveolar lavage $\mathrm{C}-\mathrm{BAL}=$ conventional bronchoalveolar lavage.

Gold standard: blind open lung biopsies

The best sensitivities for P-BAL samples were obtained using a threshold of $5 \%$ and $10 \%$ in both patients who had and had not received antibiotics (58\% each). Calculated specificities were $100 \%$. For C-BAL samples a cutoff point of $2 \%$ was the most accurate (sensitivity $67 \%$, specificity $100 \%$ ) in patients who had not been treated with antibiotics, while in those who had received antibiotics the specificity decreased to $67 \%(\mathrm{p}<0.0001)$.

When the diagnostic value parameters obtained using bronchoscopically guided biopsies and blind biopsies as gold standards were compared, most sensitivities for P-BAL samples (both for patients with and without antibiotics and independently of the threshold chosen) were significantly higher and the specificities were invariably lower. Similar changes were seen for specificities in C-BAL specimens from patients who had not received antibiotics (tables 2 and 3).

Cell counts and extracellular microorganisms in the BAL fluid are shown in table 4 . There were no significant differences between the percentages of PMNL, macrophages, lymphocytes, and extracellular microorganisms in samples from patients who had and had not received antibiotics in both types of BAL fluid samples. All samples contained less than $1 \%$ of squamous epithelial cells.

QUANTITATIVE CULTURES: P-BAL

Gold standard: bronchoscopically guided open lung biopsy

The sensitivity was $22 \%$, specificity $76 \%$, positive predictive value $54 \%$ and negative predictive value $43 \%$. In the eight patients who were not treated with antibiotics the sensitivity of the P-BAL samples decreased significantly

Table 5 Diagnostic value of protected and conventional bronchoalveolar lavage

\begin{tabular}{lccl}
\hline & Protected $B A L$ & Conventional $B A L$ & $p$ \\
\hline Mean (SD) $\log _{10}(\mathrm{cfu} / \mathrm{ml})$ & $3.1(1 \cdot 3)$ & $3 \cdot 9(1 \cdot 8)$ & NS \\
Gold standard: bronchoscopically guided open lung biopsy: & \\
Sensitivity & $22 \%$ & $35 \%$ & $<0 \cdot 05$ \\
Specificity & $76 \%$ & $43 \%$ & $<0 \cdot 001$ \\
PPV & $54 \%$ & $43 \%$ & NS \\
NPV & $43 \%$ & $35 \%$ & NS \\
Gold standard: blind open lung biopsies: & $22 \%$ & & \\
Sensitivity & $100 \%$ & $45 \%$ & 0.0005 \\
Specificity & $100 \%$ & $55 \%$ & $<0.001$ \\
PPV & $18 \%$ & $81 \%$ & NS \\
NPV & $19 \%$ & \\
\hline
\end{tabular}

$\mathrm{PPV}=$ positive predictive value; $\mathrm{NPV}=$ negative predictive value. (from $22 \%$ to $0 \% ; \mathrm{p}<0.0001$ ). No differences were shown in specificity.

Gold standard: blind open lung biopsies

The sensitivity was $22 \%$ and specificity $100 \%$ compared with the specificity of bronchoscopically guided biopsy samples; the positive predictive value was $100 \%$ and the negative predictive value $18 \%$. In the eight patients who did not receive antibiotic treatment there were no differences in the sensitivity, specificity, and positive and negative predictive values.

QUANTITATIVE CULTURES: C-BAL Gold standard: bronchoscopically guided open lung biopsy

A sensitivity of $35 \%$, a specificity of $43 \%$, and positive and negative predictive values of $43 \%$ and $35 \%$, respectively, were obtained for CBAL samples and no differences were seen between patients who did and did not receive antibiotic therapy.

Gold standard: blind open lung biopsies

The sensitivity was $45 \%$, specificity $55 \%$, and positive predictive and negative predictive values were $81 \%$ and $19 \%$, respectively. In the eight patients who did not receive antibiotics the sensitivity of the C-BAL samples increased significantly ( $38 \%$ versus $58 \%$; $<0.0001)$; there were no differences for specificity. Table 5 shows the different diagnostic values of both types of bronchoalveolar lavage using the two different types of gold standard (bronchoscopically guided and blind pulmonary biopsies). Overall, calculated sensitivities were significantly higher with C-BAL than with PBAL samples $(35 \%$ and $45 \%$ versus $22 \%$ and $22 \% ; \mathrm{p}<0.05$ and $\mathrm{p}=0.0005$, respectively). Conversely, specificities were higher with PBAL than with C-BAL samples $(76 \%$ and $100 \%$ versus $43 \%$ and $55 \% ; p=0.00001$ and $\mathrm{p}<0.0001$, respectively).

When the diagnostic value parameters of P-BAL and C-BAL samples were considered using blind biopsy samples as the gold standard, higher specificity, higher positive predictive values, and lower negative predictive values were obtained in all cases than those obtained from bronchoscopically guided biopsies (table 5). 
AGREEMENT BETWEEN BAL CULTURES

Sixty three microorganisms were isolated from P-BAL cultures, of which 50 (79\%) were concomitantly isolated from C-BAL cultures. Conversely, 74 microorganisms were isolated from C-BAL cultures of which $50(67 \%)$ were also concomitantly isolated from P-BAL cultures. Quantitative cultures of P-BAL correlated moderately with those of C-BAL $(r=0.5, \mathrm{p}=$ $0 \cdot 02)$. Table 6 shows the number of species isolated in counts of $\geq 10^{4} \mathrm{cfu} / \mathrm{ml}$ from both $\mathrm{P}$ BAL and C-BAL fluids and the mean percentages of intracellular organisms in relation to the presence or absence of histological pneumonia and as a function of the type of biopsy sample obtained (bronchoscopically guided or blind pulmonary biopsy).

\section{RELATIONSHIP BETWEEN BAL QUANTITATIVE} CULTURES AND PNEUMONIA

The following potentially pathogenic microorganisms were isolated in counts of $\geq 10^{4} \mathrm{cfu} /$ $\mathrm{ml}$ from P-BAL fluid cultures corresponding with histological pneumonia in bronchoscopically guided biopsies: Pseudomonas aeruginosa (4), Staphylococcus aureus (1), Xanthomonas maltophilia (1), and Candida albicans (1). The microorganisms isolated in counts of $\geq 10^{4} \mathrm{cfu} / \mathrm{ml}$ from C-BAL cultures from lungs with histological pneumonia were: Pseudomonas aeruginosa (3), Staphylococcus aureus (1), Xanthomonas maltophilia (1), Serratia marcesans (1), Pseudomonas putida (1), Candida albicans (1), Enterococcus faecalis (1), and Proteus mirabilis (1).

\section{HISTOLOGY AND MICROBIOLOGY OF LUNG BIOPSIES}

Twenty five of 47 (53\%) pulmonary biopsy specimens obtained showed histological signs of pneumonia compared with 174 of 375 (46\%) of the blind pulmonary biopsy specimens. Bronchiolitis was seen in 33 samples (9\%) from 13 patients.

Broncoscopically guided biopsies yielded positive cultures in 36 of $47(76 \%)$ specimens. In those samples with histological evidence of pneumonia $(\mathrm{n}=25)$, cultures were $\geq 10^{3} \mathrm{cfu} / \mathrm{g}$ in seven biopsies, $\leq 10^{3} \mathrm{cfu} / \mathrm{g}$ in 13 biopsies, and negative in five. Blind biopsies yielded positive bacterial cultures on 279 of 375 (74\%) occasions. In those samples with histological evidence of pneumonia $(n=174)$ cultures were above the threshold of $10^{3} \mathrm{cfu} / \mathrm{g}$ in 71 cases
$(41 \%),<10^{3} \mathrm{cfu} / \mathrm{g}$ in $67(38 \%)$, and negative in $36(21 \%)$. Culture of bronchoscopically guided biopsies of P-BAL and C-BAL samples were similar in 30 of $47(64 \%)$ studies. Biopsy cultures coincided with P-BAL cultures in 34 of $47(72 \%)$ cases and with C-BAL in $36(76 \%)$. In seven studies $(15 \%)$ there was no agreement in culture results with either technique.

We found moderate correlations between quantitative cultures of coincident microorganisms isolated from bronchoscopically guided lung biopsies and quantitative cultures of P-BAL $(r=0.55, \mathrm{p}<0.005)$ or $\mathrm{C}-\mathrm{BAL}$ samples $(r=0.57, \mathrm{p}<0.005)$.

\section{Discussion}

The main finding of this study is that the presence of intracellular bacteria in the PMNLs or macrophages of the centrifuged fluid from P-BAL $(\geq 5 \%)$ or C-BAL $(\geq 2 \%)$ samples is a reasonable diagnostic marker of ventilator associated pneumonia. However, these results are influenced by prior antibiotic treatment (lower sensitivity and specificity). ${ }^{1920}$

The diagnosis of ventilator associated pneumonia still remains elusive. An important issue is that the results of quantitative culture are delayed for at least three days, resulting in empirical antibiotic treatment in the interim. Given the fact that clinical features of pneumonia in mechanically ventilated patients are neither specific nor sensitive, rapid markers of pulmonary infection might be of great value to the clinician to help prescribe an antibiotic regimen. Several markers have been reported in the literature. The direct examination for the presence of elastin fibres may help in the diagnosis of necrotising pneumonias. ${ }^{821}$ Other markers in respiratory secretions such as lactate dehydrogenase and endotoxin are being investigated. ${ }^{67}$

The percentage of intracellular organisms in the PMNLs and macrophages of the recovered BAL fluid deserves particular attention. Initial studies from the same group ${ }^{522}$ found that the use of this technique on C-BAL samples has a reasonable sensitivity and $100 \%$ specificity for the diagnosis of ventilator associated pneumonia. Yet, there was discrepancy in the threshold for differentiating the presence of pneumonia. More investigations using P-BAL samples ${ }^{10}$ were very sensitive and specific when choosing $2 \%$ intracellular organisms as a cutoff point.

Table 6 Numbers of lungs with and without pneumonia $(n=47)$, numbers of isolates of bacterial species from BAL fluids in significant counts $\left(\geq 10^{4} \mathrm{cfu} / \mathrm{ml}\right)$, and percentages of intracellular microorganisms

\begin{tabular}{|c|c|c|c|c|}
\hline \multirow[t]{2}{*}{ Pneumonia* } & \multicolumn{2}{|c|}{ No. of species $\left(\geq 10^{4} \mathrm{cfu} / \mathrm{ml}\right)$} & \multicolumn{2}{|c|}{ Mean (range) intracellular organisms (\%) } \\
\hline & $P-B A L$ & $C-B A L$ & $P-B A L$ & $C-B A L$ \\
\hline \multicolumn{5}{|c|}{$\begin{array}{l}\text { Bronchoscopically guided } \\
\text { open lung biopsies: }\end{array}$} \\
\hline $\begin{array}{cc}\text { Yes } & 25 \\
\text { No } & 22\end{array}$ & $\begin{array}{l}6 \\
5\end{array}$ & $\begin{array}{l}10 \\
10\end{array}$ & $\begin{array}{r}7(0-40) \\
15(0-90)\end{array}$ & $\begin{array}{l}11(0-80) \\
19(0-90)\end{array}$ \\
\hline \multicolumn{5}{|c|}{$\begin{array}{l}\text { Lungs (at least one positive blind } \\
\text { open lung biopsy per lung): }\end{array}$} \\
\hline $\begin{array}{ll}\text { Yes } & 40 \\
\text { No } & 7\end{array}$ & $\begin{array}{r}13 \\
3\end{array}$ & $\begin{array}{r}19 \\
3\end{array}$ & $\begin{array}{l}10(0-90) \\
0 \cdot 6(0-4)\end{array}$ & $\begin{array}{r}17(0-90) \\
2(0-12)\end{array}$ \\
\hline
\end{tabular}

* Presence or absence of histological pneumonia (bronchoscopically guided lung biopsy). 
Other investigators have confirmed the value of the detection of intracellular organisms ${ }^{91223}$ and have suggested $5 \%$ of PMNLs containing intracellular organisms as the ideal threshold to differentiate between the presence and absence of pneumonia. However, recent publications ${ }^{24}$ could not find an adequate cutoff point and the effect of antibiotic treatment was not specifically explored in these studies. Moreover, the gold standards used were limited to the presence of clinical signs of pneumonia or to the bacteriological cultures of protected specimen brush or BAL fluid only. When the histological results of blind necroscopic open lung biopsy specimens were used as a gold standard we found that the presence of $\geq 5 \%$ of PMNLs with intracellular organisms in PBAL samples or $\geq 2 \%$ in C-BAL samples was a sensitive (approximately 60\%) and very specific $(100 \%)$ tool for detecting the existence of pneumonia.

There are, however, some findings and limitations of our study that need to be emphasised. Firstly, the high specificity of intracellular organism detection found in our study is conditioned by the high prevalence of histological pneumonia (in blind open lung biopsy specimens) found in our deceased critically ill patients. Secondly, the diagnostic value parameters of intracellular organisms, particularly the sensitivity, were strongly influenced by prior antibiotic treatment. This has been reported by others ${ }^{11}{ }^{12}$ who showed a reduction in the predictive accuracy of intracellular organisms in detecting ventilator associated pneumonia in patients receiving or having recently received antibiotics. This finding probably does not apply to all patients and depends upon the intrinsic characteristics of each antibiotic (intracellular penetration, accumulation, and disposition). ${ }^{25}$ Thirdly, the diagnostic value of intracellular organism detection was generally independent of the type of BAL fluid used, although with C-BAL samples a lower threshold of intracellular organisms ( $\geq 2 \%$ ) was enough to differentiate between the presence and absence of pneumonia in patients not receiving antibiotics. However, our results could have been biased as, due to design constraints, P-BAL sampling was always performed before C-BAL sampling in each lung of our cadaveric model without evaluating an order effect. This was because the main aim of our study was to explore the results of P-BAL. Fourthly, the diagnostic value of intracellular organisms was altered by the type of gold standard used - bronchoscopically guided open lung biopsy specimens or multiple blind open lung specimens. The diagnostic value of intracellular organisms for both types of BAL samples were more specific when the analysis was taken into consideration lung-bylung and in the presence of histological pneumonia if at least one of the multiple blind open lung biopsy specimens was compatible with it. What may occur in ventilator associated pneumonia is that, once foci of pneumonia develop, infected secretions may be redistributed to other bronchopulmonary segments, possibly enhanced by the volume and flow patterns generated by the ventilator. This is why positive microbiological results (intracellular organisms or quantitative cultures) could be shown when anatomical areas, distant from the histological foci of the pneumonia, were explored and also explains why blind techniques, as other investigators have pointed out, may have similar results to bronchoscopic techniques. ${ }^{26-29}$ The presence of bronchiolitis could also partly explain the false positive results, ${ }^{14}$ although the incidence of bronchiolitis in our biopsies was small (9\%). The correlation between quantitative cultures of BAL fluid and the percentage of intracellular organisms $(r=$ 0.45 ) indicates that the detection of intracellular organisms is only partly dependent $(20 \%)$ on the lung bacterial burden. A further limitation is that, as an experimental study, the detection of intracellular organisms is restricted to BAL fluid from patients with ventilator associated pneumonia. Whether this technique may be extended to other respiratory samples such as endotracheal aspirates has not been investigated, although it seems that the most adequate sample for processing is the $\mathrm{BAL}$ fluid. Finally, a potential pitfall of this study could be the inclusion of gold standard biopsy samples with histological pneumonia and negative cultures. This only occurred in $20 \%$ of lung samples with histological evidence of pneumonia, although our results did not alter when these samples were excluded from the analysis.

Overall, quantitative cultures of C-BAL yielded both a poor sensitivity and specificity, irrespective of the gold standard (bronchoscopically guided or multiple blind samples) used. This reinforces previously published work by our group using a single necroscopic bronchoscopically guided open lung biopsy specimen as a gold standard. ${ }^{30}$ Quantitative cultures of P-BAL were less sensitive and much more specific than those obtained with C-BAL, confirming the lower contamination with P-BAL.

Meduri et $a l^{10}$ found that P-BAL cultures had a high sensitivity and specificity for diagnosing ventilator associated pneumonia. However, our results showed a much lower sensitivity. This can be explained by differences in the gold standard used (histological results in our study), the type of study population (immediately deceased patients in our case), prior antibiotic treatment (only 48 hours without antibiotic treatment in those cases considered not previously treated), exclusion of nonpathogenic microorganisms, and the level of threshold chosen $\left(10^{4} \mathrm{cfu} / \mathrm{ml}\right)$. These explanations can also be applied to the lower sensitivity obtained with C-BAL cultures compared with previous studies. ${ }^{22}$

Differential neutrophil counts and the presence of extracellular bacteria in P-BAL or CBAL samples did not help to differentiate cases of pneumonia in whom prior antibiotic treatment had or had not been given - a finding previously outlined by other groups. ${ }^{22}$ However, Meduri et $a l^{10}$ have shown that Gram stains of P-BAL fluids were positive in all their cases of pneumonia, thus allowing for early and accurate diagnosis of lower respiratory tract 
infections before the results of cultures were available. Other investigators ${ }^{9124}$ have reported that the percentage of neutrophils in the lavage fluid is greater in cases of pneumonia, yet no differences in the percentage of macrophages or lymphocytes were shown. ${ }^{9}$ Differences among studies have to be explained by differences in the underlying diseases of the study populations.

Supported by grant $94 / 0583 \& 95 / 1903$ from the FIS (Fondo de Investigación Sanitaria). Comissionat Per Universitats i Recerca de la Generalitat de Catalunya (GRQ94-9103).

1 Fagon JY, Chastre J, Domart Y, Trouillet JL, Pierre J, Darne C, et al. Nosocomial pneumonia in patients receiving , et al. Nosoconial pnetation: prospective analysis of continuous mech anical ventilation: prospective analysis of quantitative culture techniques. Am Rev Respir Dis 1989; quantitative

2 Torres A, Aznar R, Gatell JM, Jiménez P, González J, Ferrer $A$, et al. Incidence, risk and prognosis factors of nosocomial pneumonia in mechanically ventilated patients. $A m$ Rev Respir Dis 1990;142:523-8.

3 Craven DE, Kunches LM, Lichtenberg DA, Kollisch NR Barry MA, Heeren TC. Nosocomial infection and fatality in medical and surgical intensive care unit patients. Arch Intern Med 1988;148:1161-8.

4 El-Ebiary M, Torres A, González J, Puig de la Bellacasa J, Garcia C, Jimenez de Anta, et al. Quantitative cultures of endotracheal aspirates for the diagnosis of ventilatorassociated pneumonia. Am Rev Respir Dis 1993;148:15527 .

5 Chastre J, Fagon JY, Soler P, Domart Y, Pierre J, Dombret MC. Quantification of BAL cells containing intracellular bacteria rapidly identifies ventilated patients with nosocomial pneumonia. Chest 1989;95:190S-2S.

6 Pugin J, Auchenthaler R, Delaspre O, Van Gessel E, Suter PM. Rapid diagnosis of Gram negative pneumonia by assay of endotoxin in BAL fluid. Thorax 1992;47:547-9.

7 Efferen $\mathrm{LS}, \mathrm{Ng} \mathrm{R}$, Palak D. Utility of sputum lactate dehydrogenase levels in diagnosing acute infectious bronchopulmonary disease. Am 7 Respir Crit Care Med 1994 149:A340.

8 Salata R, Lederman MM, Shlaes DM, Jacobs MR, Eckstein $\mathrm{E}$, Tweardy $\mathrm{D}$, et al. Diagnosis of nosocomial pneumonia in intubated, intensive care unit patients. Am Rev Respir Dis 1987;135:426-32.

9 Pugin J, Auckenthaler R, Nabil M, Janssens JP, Lew PD, Suter PM. Diagnosis of ventilator-associated pneumonia by bacteriological analysis of bronchoscopic and nonbronchoscopic "blind" bronchoalveolar lavage fluid. $\mathrm{Am}$ Rev Respir Dis 1991;143:1121-9.

10 Meduri GU, Beals DH, Maijub AG, Baselski V. Protected bronchoalveolar lavage. A new bronchoscopic technique to retrieve uncontaminated distal airway secretions. $A n$ Rev Respir Dis 1991;143:855-64.

11 Dotson RG, Pingleton SK. The effect of antibiotic therapy on recovery of intracellular bacteria from bronchoalveolar lavage in suspected ventilator-associated pneumonia. Chest 1993;103:541-6.

12 Vallés J, Rello J, Fernández R, Blanch LL, Baigorri F, Mestre $\mathrm{J}$, et al. Role of bronchoalveolar lavage in mechanically $\mathrm{J}$, et al. Role of bronchoalveolar lavage in mechanically Microbiol Infect Dis 1994;13:549-58.
13 Meduri GU, Chastre J. The standardization of bronchoscopic technique for ventilator-associated pneumonia. Chest 1992;102:557-64S.

14 Fábregas N, Torres A, El-Ebiary M, Ramírez J, Hernández C, González J, et al. Histopathological and microbiological aspects of ventilator-associated pneumonia. Anesthesiology 1996 (in press).

15 Balows A, Hausler jr WJ, Herrmann KL, Isenberg HD Shadomy HS, eds. Manual of clinical microbiology. 5th edn, Section III. Washington, DC: American Society for Microbiology; 1991

16 Norusis MJ. SPSSPC + statistics V 4.0. Statistical Package for Social Sciences. SPSS/Inc. 1990.

17 Griner PF, Mayewski RJ, Mushlin AI, Grunland P. Selection and interpretation of diagnostic tests and procedures. Ann Intern Med 1981;94:533-600.

18 Sackett DL, Haynes RB, Guyatt GH, Tugwell P. The interpretation of the diagnostic data. In: Sacket DL, Haynes RB, Guyatt GH, Tugwell P, eds. Clinical epidemiology. A basic science for clinical medicine. Boston: Little Brown, 1992:69-152.

19 Niederman MS, Torres A, Summer W. Invasive diagnostic testing is not needed routinely to manage ventilator-associated pneumonia. Am $\mathcal{F}$ Respir Crit Care Med 1994;150. 565-9.

20 Chastre J, Fagon JY. Invasive diagnostic testing should be routinely used to manage ventilated patients with suspected pneumonia. Am $\mathcal{F}$ Respir Crit Care Med 1994;150 570-4.

21 El-Ebiary M, Torres A, Gonzalez J, Martos A, Puig de la Bellacasa J, Ferrer M, et al. Use of elastin fibre detection in the diagnosis of ventilator associated pneumonia. Thorax 1995;50:14-17.

22 Chastre J, Fagon JY, Soler P, Bornet M, Domart Y, Trouillet $J \mathrm{Y}$, et al. Diagnosis of nosocomial bacterial pneumonia in intubated patients undergoing ventilation: comparison of the usefulness of bronchoalveolar lavage and the protected the usefulness of bronchoalveolar lavage and the pr

23 Solé-Violán J, Rodriguez de Castro F, Rey A, MartínGonzalez, Cabrera-Navarro P. Usefulness of microscopic examination of intracellular organisms in lavage fluid in ventilator-associated pneumonia. Chest 1994;106:889-94.

24 Aubas S, Aubas P, Capdevila X, Darbas H, Roustan JP, Du Cailar J. Bronchoalveolar lavage for diagnosing bacterial pneumonia in mechanically ventilated patients. $A m$ F Respir Crit Care Med 1994;149:860-6.

25 Tulkens PM. Intracellular distribution and activity of antibiotics. Eur f Clin Microbiol Infect Dis 1991;10:100-6.

26 Pham LH, Brun-Buisson C, Legrand P, Rauss A, Verra F, Brochard L, et al. Diagnosis of nosocomial pneumonia in mechanically ventilated patients. Comparison of a plugged telescoping catheter with the protected specimen brush. Am Rev Respir Dis 1991;143:1055-61.

27 Rouby JI, Martin de Lassale EM, Poete P, Nicolas MH Bodin L, Jarlier V, et al. Nosocomial bronchopneumonia in the critically ill. Am Rev Respir Dis 1992;146:1059-66.

28 Jordá R, Parras F, Ibañez J, Reina J, Bergadá J, Raurich JM. Diagnosis of nosocomial pneumonia in mechanically ventilated patients by the blind protected telescoping catheter. Intensive Care Med 1993;19:377-82.

29 Torres A, Puig de la Bellacasa J, Rodriguez-Roisin R Jiménez de Anta $M^{a} T$, Agusti-Vidal A. Diagnostic value of telescoping plugged catheters in mechanically ventilated patients with bacterial pneumonia using the Métras catheter. Am Rev Respir Dis 1988;138:117-20.

30 Torres A, El-Ebiary M, Padró L, González J, Puig de la Bellacasa J, Ramirez J, et al. Validation of different techniques for the diagnosis of ventilator-associated pneumonia. Comparison with immediate postmortem pulmonary biopsy. Am f Respir Crit Care Med 1994;149. 324-31. 